\title{
Experiencia en procedimientos laparoscópicos en México durante 2015: ¿dónde estamos?
}

\author{
Laparoscopic procedures experience in Mexico during 2015: ¿Where are we?
}

\author{
Gerardo Chávez-Saavedra', Elia Lara-Lona ${ }^{1 *}$, Carlos Hidalgo-Valadez ${ }^{1}$, Nicolás Romero-Salinas ${ }^{1}$ y \\ Gonzalo de J. Méndez-Sashida ${ }^{2}$ \\ ${ }^{1}$ Departamento de Medicina y Nutrición, Universidad de Guanajuato; 2Servicio de Cirugía General, Unidad Médica de Alta Especialidad del Bajío, \\ Instituto Mexicano del Seguro Social. Guanajuato, México
}

\section{Resumen}

Antecedentes: Han pasado 26 años desde la primera colecistectomía laparoscópica en México. Desde entonces, la laparoscopía se ha adoptado de forma variable y se ha extendido a diferentes especialidades. Objetivo: Identificar el sitio que ocupan los abordajes laparoscópicos en México. Método: Se buscaron los códigos que tuvieran la palabra laparoscopia o laparoscópica en los registros del Sistema Automatizado de Egresos Hospitalarios en 2015. Con los datos obtenidos se realizó un estudio descriptivo y retrospectivo. Resultados: Se encontraron 55 procedimientos diferentes en un total de 30,174. De ellos, el $79.7 \%$ en mujeres y el $20.3 \%$ en hombres. La edad más común fue entre los 25 y 29 años. Los diez primeros fueron colecistectomía, apendicectomía, histerectomía total abdominal laparoscópica, procedimientos para creación de competencia esfinteriana esofagogástrica, salpingo-ooforectomía unilateral, colecistectomía parcial, escisión local o destrucción de ovario, plastia umbilical, apendicectomía incidental y ooforectomía unilateral (94.6\% del total). Los Estados con el mayor número reportado fueron Ciudad de México, Estado de México, Jalisco, Guanajuato y Sonora. Conclusiones: Los procedimientos laparoscópicos han ido en aumento en los sistemas de salud nacionales y hay concordancia de los más comunes con las estadísticas internacionales. Sin embargo, es necesario diversificarlos y disminuir los tiempos de estancia hospitalaria. Su aplicación está en proceso y aún enfrentan retos en relación con la disponibilidad de elementos organizacionales, equipo, infraestructura y entrenamiento, aunque existen diferentes alternativas para vencerlos.

PALABRAS CLAVE: Cirugía laparoscópica. Laparoscopía. Procedimientos laparoscópicos.

\section{Summary}

Background: 26 years have passed from the first laparoscopic cholecystectomy in Mexico. Since then the laparoscopy has been adopted of variable way and has extended into different specialties. Objective: To identify the place that laparoscopic approaches occupy at the present time in Mexico. Method: We searched the codes that had the word laparoscopy or laparoscopic in the records of the Automated System of Hospitable Discharges in 2015. Based on the obtained information there was realized a descriptive and retrospective study. Results: We found 55 different procedures in a total of 30,174. Of them, $79.7 \%$ in women and $20.3 \%$ in men. The most common age was between 25 and 29 years. The ten first ones were cholecystectomy, appendectomy, total abdominal laparoscopic hysterectomy, procedures to create esophago-gastric sphincter competence, unilateral salpingo-oophorectomy, partial cholecystectomy, ovarian resection, umbilical hernia repair, incidental appen-

\footnotetext{
Correspondencia:

*Elia Lara-Lona

Av. Puente del Milenio, 1001

Fracc. San Carlos León

Guanajuato, Gto., México

E-mail: elia.lara@ugto.mx
}

Fecha de recepción: 28-06-2018

Fecha de aceptación: 20-09-2018

DOI: 10.24875/CIRU.18000562

Cir Cir. 2019;87:292-298

Contents available at PubMed

www.cirugiaycirujanos.com 
dectomy and unilateral oophorectomy (94.6\%). The States with the major number are Mexico City, State of Mexico, Jalisco, Guanajuato and Sonora. Conclusions: The laparoscopic procedures have increase in the national health systems and there is concordance of the most common with the international statistics. However, is necessary to diversify them and reduce the times of hospital stay. Its application is in process and it still face challenges in relation to availability of organizational elements, equipment, infrastructure and training, although there are different ways to overcome them.

KEY WORDS: Laparoscopic procedures. Laparoscopic surgery. Laparoscopy.

\section{Introducción}

Han pasado 33 años desde la primera colecistectomía laparoscópica realizada en el mundo por el Dr. Mühe en Alemania', 31 años desde la primera efectuada por el Dr. Mouret en Francia y 28 años desde la primera colecistectomía laparoscópica en México y América Latina por el Dr. Leopoldo Gutiérrez². Desde entonces, la laparoscopía se ha extendido en todo el país y se ha diversificado en diferentes especialidades. Se ha utilizado por diferentes indicaciones y en diferentes diagnósticos ${ }^{3}$. Se ha obtenido experiencia suficiente y el número de complicaciones es menor, lo que se refleja claramente en la curva de aprendizaje ${ }^{4}$.

El caso más representativo es el de la colecistectomía laparoscópica, porque actualmente ha sido aceptada mundialmente como el tratamiento de elección para la litiasis vesicular. Ello es debido a los ya demostrados beneficios del abordaje laparoscópico: incisiones más pequeñas, menor dolor posoperatorio, estancia hospitalaria más corta (ambulatoria en algunos centros), retorno más rápido a las actividades cotidianas, menores morbilidad y mortalidad al ser efectuada por personal entrenado, y menor costo hospitalario (aunque el procedimiento quirúrgico por sí solo se eleve) $)^{5}$.

Todo lo anterior se puede aplicar en forma general a cualquier otro procedimiento efectuado por abordaje laparoscópico en manos expertas. Ejemplos de ellos son el trauma abdominal penetrante y no penetrante ${ }^{6}$, y las emergencias abdominales no traumáticas (apendicitis aguda, obstrucción intestinal, hernia interna, perforación de la vesícula biliar, isquemia mesentérica, úlcera péptica, diverticulitis) ${ }^{7}$. Los resultados en general muestran que la laparoscopía es segura, eficaz y costo-efectiva comparada con la cirugía abierta en diversos procedimientos y de diferentes especialidades; incluso se sugiere que debe ser de elección en todo procedimiento común, independientemente de la gravedad de la enfermedad ${ }^{8}$. Existe suficiente experiencia en procedimientos ginecológicos ${ }^{9}$, así como guías para su uso en el diagnóstico y el tratamiento de problemas quirúrgicos durante el embarazo ${ }^{10}$.
La laparoscopía, mediante la cirugía de corta estancia, representa una opción para enfrentar la creciente demanda de atención médica ante una capacidad de hospitalización limitada ${ }^{11,12}$. De hecho, se ha encontrado una alta preferencia por este tipo de cirugía en pacientes con condiciones benignas ${ }^{13}$. Para las neoplasias malignas, como el cáncer de endometrio, ofrece índices más bajos de morbilidad y una recuperación más rápida de acuerdo con lo reportado en el Hospital de Oncología del Centro Médico Nacional Siglo XXI, aunque el pronóstico depende más de la etapa de la enfermedad que del abordaje $e^{14}$.

Todo ello hace necesario identificar el lugar que la laparoscopía ocupa en los procedimientos quirúrgicos actuales, en relación con su impacto en los sistemas de salud, para entonces saber hacia dónde orientarla. El objetivo es analizar los registros de los procedimientos laparoscópicos en México, en 2015, por sexo, edad, entidad federativa, procedimientos e indicaciones más comunes, estancia y tipo de anestesia, y posteriormente interpretarlos para comprender el panorama actual y compararlos con las estadísticas internacionales para establecer algunas consideraciones que contribuyan a un plan estratégico de mejora, a fin de aprovechar sus ventajas en el contexto de nuestro propio Sistema Nacional de Salud. De igual forma, generar nuevas líneas de investigación sobre procedimientos de mínima invasión, como abordajes laparoscópicos en procedimientos diagnósticos.

\section{Método}

Se realizó una búsqueda de los códigos que tuvieran la palabra «laparoscopia» o «laparoscópica» en la Clasificación Internacional de Enfermedades 9a revisión, modificación clínica. Lista Tabular Clasificación de Procedimientos (CIE 9 MC) Lista Tabular del Centro Mexicano para la Clasificación de Enfermedades y Centro Colaborador para la Familia de Clasificaciones Internacionales de la OMS en México (CEMECE) para identificar todos aquellos procedimientos registrados en el Sistema Automatizado de Egresos Hospitalarios 


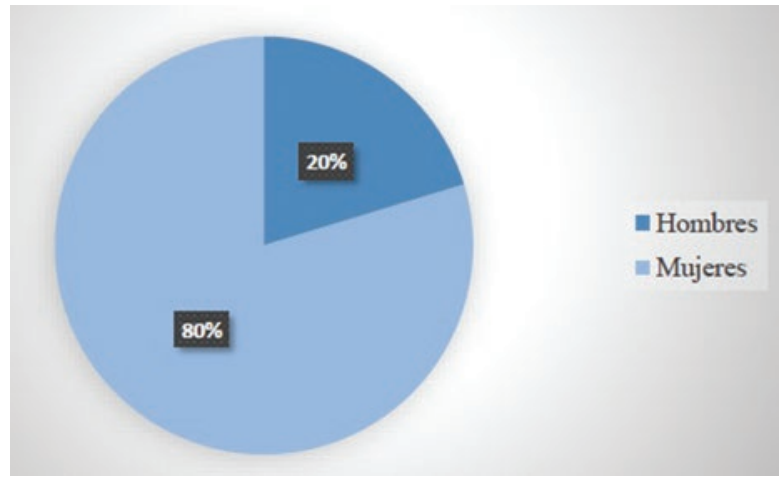

Figura 1. Procedimientos laparoscópicos en México, 2015.

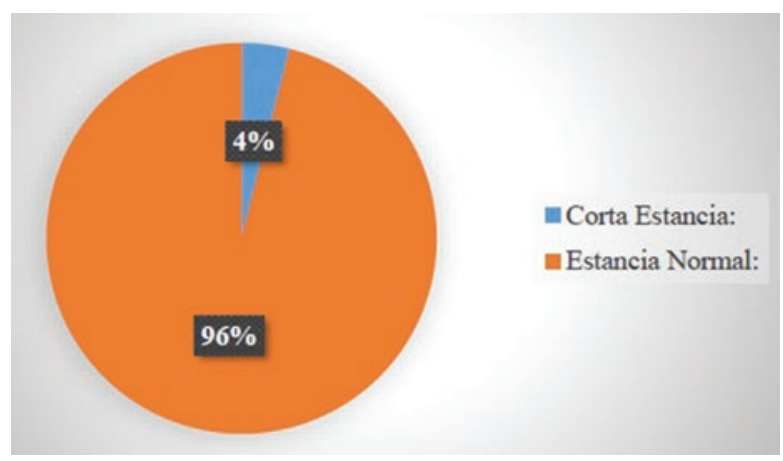

Figura 2. Tiempo de estancia posoperatoria.

durante el año 2015. Dicho sistema se encuentra disponible de manera pública en la página www.salud. gob.mx/cubos. A partir de los datos obtenidos se realizó un estudio descriptivo y retrospectivo. Se incluyeron todos aquellos procedimientos quirúrgicos, y por tanto se excluyeron los de tipo diagnóstico.

\section{Resultados}

Este estudio encontró 55 diferentes procedimientos de cirugía general, ginecología y urología realizados a través de abordajes laparoscópicos. Se realizaron durante 2015 un total de 30,174 procedimientos laparoscópicos; de ellos, el $79.7 \%$ en mujeres y el $20.3 \%$ en hombres (Fig. 1). La edad más común en que fueron realizados osciló entre los 25 y los 29 años. Los diez primeros procedimientos fueron colecistectomía, apendicectomía, histerectomía total abdominal laparoscópica, procedimientos para creación de competencia esfinteriana esofagogástrica, salpingo-ooforectomía unilateral, colecistectomía parcial, escisión local o destrucción de ovario, plastia umbilical, apendicectomía incidental y ooforectomía unilateral. La colecistectomía laparoscópica representa el $75.9 \%$. En conjunto estos diez procedimientos corresponden al $94.6 \%$ del total de los procedimientos encontrados (Tabla 1).
Tabla 1. Principales procedimientos laparoscópicos en México, 2015

\begin{tabular}{|c|c|c|}
\hline & $\mathbf{N}$ & $(\%)$ \\
\hline 1. Colecistectomía laparoscópica & 22,921 & $(75.96)$ \\
\hline 2. Apendicectomía laparoscópica & 2317 & $(7.67)$ \\
\hline 3. Histerectomía total abdominal laparoscópica & 768 & $(2.54)$ \\
\hline $\begin{array}{l}\text { 4. Otros procedimientos para la creación de } \\
\text { competencia esfinteriana esofagogástrica }\end{array}$ & 663 & $(2.19)$ \\
\hline $\begin{array}{l}\text { 5. Salpingo-ooforectomía unilateral } \\
\text { laparoscópica }\end{array}$ & 459 & $(1.52)$ \\
\hline 6. Colecistectomía parcial laparoscópica & 354 & $(1.17)$ \\
\hline $\begin{array}{l}\text { 7. Otra escisión local o destrucción } \\
\text { laparoscópica de ovario }\end{array}$ & 305 & $(1.01)$ \\
\hline 8. Reparación laparoscópica de hernia umbilical & 248 & $(0.82)$ \\
\hline 9. Apendicectomía incidental laparoscópica & 180 & $(0.59)$ \\
\hline 10. Ooforectomía unilateral laparoscópica & 171 & $(0.56)$ \\
\hline $\begin{array}{l}\text { 11. Reparación laparoscópica de hernia inguinal } \\
\text { directa con injerto o prótesis }\end{array}$ & 161 & $(0.53)$ \\
\hline Total & 28,547 & $(94.56)$ \\
\hline
\end{tabular}

El $96 \%$ tuvieron estancia normal y el $4 \%$ fueron de corta estancia (Fig. 2); el $79.35 \%$ oscilaron entre los 0 y 3 días de estancia. Por lo tanto, el $20.65 \%$ permaneció más de 3 días en hospitalización posoperatoria. El procedimiento más común ofrecido como corta estancia fue la colecistectomía, seguido de la plastia inguinal y de los procedimientos para creación de competencia esfinteriana esofagogástrica. El 98\% fueron egresados por mejoría o curación. Los Estados con mayor número registrado fueron Ciudad de México, Estado de México, Jalisco, Guanajuato y Sonora (Tabla 2).

\section{Discusión}

Haciendo una interpretación de los resultados se puede obtener una visión general del estado de los abordajes laparoscópicos para eventos quirúrgicos (no diagnósticos) en México en un momento determinado (año 2015). La laparoscopia para procedimientos diagnósticos no se estudia aquí porque representa una línea de investigación independiente. La intención es ofrecer una referencia para compararla con otros años y obtener una comprensión dinámica de su comportamiento. Al conocer el rumbo de los abordajes laparoscópicos, se facilita el empoderamiento del cirujano como agente participativo en la gestión de políticas públicas en salud, a través de la apropiación, 
Tabla 2. Estados con el mayor número de procedimientos laparoscópicos

\begin{tabular}{lc}
\hline Entidad & $\mathbf{N} .^{0}$ procedimientos \\
\hline 1. Distrito Federal & 8162 \\
2. Estado de México & 2016 \\
3. Jalisco & 2933 \\
4. Guanajuato & 1850 \\
5. Sonora & 998 \\
\hline
\end{tabular}

la adecuación y el aprovechamiento de la laparoscopia en el sistema de salud de México.

Primero es necesario seleccionar indicadores de gestión hospitalaria pertinentes para evaluar las cirugías a través de abordajes laparoscópicos o adaptar los que se usan comúnmente para evaluar la calidad hospitalaria (índice de rotación, porcentaje de ocupación, etc.). La comparación de estos indicadores, una vez conocidos, con los estándares utilizados internacionalmente, permite mejorar la toma de decisiones gracias a una adecuada gestión de la información y su transformación en conocimiento ${ }^{15}$. El resultado es un cambio, primero de paradigma y luego de la estructura institucional del sistema de salud nacional hacia el cumplimiento de objetivos más acordes con su visión y misión, donde la laparoscopia puede desempeñar un papel fundamental. Es así como el cirujano puede contribuir con el mayor reto del actual sistema de salud: contar con alternativas para favorecer su integración con el fin de ofrecer el ejercicio universal e igualitario del derecho a la protección de la salud ${ }^{16}$.

El análisis de los resultados muestra que aún existe poca diversificación en el uso de los abordajes laparoscópicos entre los 55 diferentes procedimientos registrados: el $94.6 \%$ se usan en solo diez procedimientos. Es probable que la explicación se deba a múltiples factores: falta de entrenamiento y experiencia en los diferentes procedimientos, falta de disponibilidad de equipo, patologías y procedimientos menos frecuentes, etc. Es un punto crítico que requiere acciones correctivas dentro de un plan estratégico más amplio.

Resulta evidente que las mujeres son las más beneficiadas, al constituir prácticamente el $80 \%$ del total de los procedimientos. Lo anterior es similar a lo reportado en otras series nacionales e internacionales, al menos en lo relativo a la colecistectomía ${ }^{5,17,18}$. Ello se explica por la mayor incidencia de litiasis vesicular en ellas, así como por los diferentes procedimientos ginecológicos realizados.
En concordancia con las estadísticas internacionales, en las que el $80 \%$ de las colecistectomías se realizan por vía laparoscópica ${ }^{17-19}$, en México el procedimiento laparoscópico más común sigue siendo la colecistectomía, seguida por la apendicectomía. Ambas afecciones son muy comunes para el cirujano general, y se aprovechan ventajas como la disminución de la estancia hospitalaria, de la morbilidad y del dolor posoperatorio ${ }^{19,20}$.

En el caso de la patología herniaria, también común para el cirujano general, la laparoscopia se ha adoptado de forma menos consistente. Resulta interesante observar, por un lado, que de acuerdo con lo reportado la plastia umbilical supera a la inguinal; esta ocupa el undécimo lugar a pesar de que es mucho más común que la umbilical21. La causa probable es la inconsistencia en los registros. Por otro lado, la dificultad técnica, el uso de equipo laparoscópico y el aumento en el tiempo quirúrgico (que incrementan los costos) pueden ser los motivos que han enlentecido su adopción, sobre todo en la hernia umbilical, para la que resulta técnicamente y económicamente más asequible por abordaje abierto ${ }^{22,23}$.

Durante el año 2015 se hizo una estimación de los indicadores de efectividad, eficiencia y pertinencia para determinar el desempeño de los hospitales en México ${ }^{24}$. Para la eficiencia se tomó, entre otros indicadores, el porcentaje de hernioplastias resueltas como cirugía de corta estancia (frecuencia relativa de pacientes a los que se realizó una reparación de hernia y cuya estancia hospitalaria fue menor de 24 horas, con respecto al total de egresos a los que se practicó una hernioplastia). Aquí cabría considerar la hernioplastia por abordaje laparoscópico también como cirugía de corta estancia. Aunque el tiempo de estancia hospitalaria es mayor, esta diferencia es mínima (0.1 días) y podría considerarse insignificante ${ }^{23}$.

El porcentaje de pacientes que permanecen en estancia normal sigue siendo muy alto $(96 \%)$ a pesar de que ya se ha demostrado la conveniencia de la corta estancia en varios procedimientos y en pacientes bien seleccionados ${ }^{11}$. Probablemente se debe a la falta de elementos organizativos (personal disponible, control de procesos, etc.), a aspectos de infraestructura y a que las camas de corta estancia son no censables porque se destinan a la atención transitoria y no generan egresos hospitalarios ${ }^{25,26}$. En relación con lo último, la corta estancia no refleja la eficiencia hospitalaria al no poderse medir a través de indicadores como el índice de rotación (número de egresos en unidades de segundo nivel en un periodo 
determinado/total de camas censables) y el número total de egresos. Ello obliga a dejar pacientes hospitalizados para incrementar los índices de eficiencia durante las evaluaciones ${ }^{15}$.

De la misma forma se encontró que en ginecología se han aprovechado los beneficios de los abordajes laparoscópicos en varios procedimientos. La histerectomía total abdominal, la salpingo-ooforectomía unilateral, la escisión local o destrucción de ovario, y la ooforectomía unilateral son cuatro de los diez procedimientos laparoscópicos más frecuentes realizados en México durante 2015. En concreto, para la histerectomía como uno de los procedimientos más comunes, se ha demostrado la conveniencia del abordaje laparoscópico respecto a estancia hospitalaria, tiempo operatorio, analgesia posoperatoria y complicaciones ${ }^{26}$.

Para el resto de los procedimientos ginecológicos listados, los abordajes por laparoscopia ya se han adoptado con éxito. Se conocen las indicaciones, las contraindicaciones y las posibles complicaciones. La escisión de ovario y la salpingo-ooforectomía generalmente se usan en el contexto de patología quística, embarazo ectópico y procesos infecciosos agudos ${ }^{27}$.

No hay procedimientos urológicos con abordaje laparoscópico dentro de los diez más frecuentes, pero existe evidencia de la experiencia que se ha ganado en la nefrectomía laparoscópica de acuerdo con lo reportado en la literatura nacional, tanto en abordajes lumboscópicos como transperitoneales. Las indicaciones han sido comúnmente el tratamiento de tumores renales confinados y la patología renal hipofuncionante, y resulta una alternativa a la cirugía abierta con menor morbilidad y mortalidad, y con el mismo resultado oncológico a largo plazo ${ }^{28}$.

La colecistectomía parcial por abordaje laparoscópico aparece en el sexto lugar probablemente porque representa una alternativa viable a la conversión o la colecistostomía en caso de una colecistectomía técnicamente difícil o en pacientes de alto riesgo. Es un procedimiento seguro y rápido que reduce la incidencia de lesión de la vía biliar y la morbimortalidad perioperatoria ${ }^{29}$.

La apendicectomía incidental laparoscópica ocupa el noveno lugar a pesar de que todavía genera controversia entre los cirujanos. En una revisión de 18 años en la que se evaluó la costo-efectividad, se concluyó que es un procedimiento que resulta seguro durante cirugías abiertas para otras patologías intestinales, con hallazgos patológicos muy bajos, pero no inexistentes (p. ej., cistoadenoma mucinoso, tumor carcinoide, etc.). Para la apendicectomía incidental laparoscópica solo se encontró beneficio en el costo en procedimientos gastrointestinales bajos por patologías benignas para hombres y mujeres por debajo de los 25 años. Si fue por una indicación de malignidad, solo tuvo costo-beneficio en hombres menores de 20 años y en mujeres menores de 15 años $^{30}$.

El incremento en la obesidad, las enfermedades metabólicas y sus complicaciones en los últimos años ${ }^{16}$ crea un nicho donde los abordajes laparoscópicos pueden diversificarse y consolidarse bajo indicaciones claras y con pacientes adecuadamente seleccionados ${ }^{31,32}$. Incluso procedimientos no metabólicos o bariátricos, como la apendicitis aguda, en pacientes con obesidad tienen resultados clínicos superiores en comparación con los abordajes abiertos ${ }^{33}$. Debido a la alta frecuencia de estos padecimientos en nuestro medio, los cirujanos debemos generar mayor evidencia a través de la investigación en esta área para lograr una legislación avanzada que autorice y cubra el tratamiento por los sistemas de salud, como ya se ha hecho en Brasil y Chile en América Latina $^{34}$.

Solo el $4.22 \%$ de los procedimientos se reportan como de corta estancia. De los diez primeros (el $92.77 \%$ ), nueve pueden realizarse en esta modalidad: colecistectomía, apendicectomía no complicada, apendicectomía incidental, ooforectomía, salpingo-ooforectomía, escisión o destrucción de ovario, histerectomía, procedimientos para creación de competencia esfinteriana esofagogástrica y plastia umbilical. Una explicación para este bajo porcentaje es que el registro de hospitalización, y no la corta estancia, incrementa la productividad de un servicio. Esto representa un área de oportunidad para los hospitales, debido a que muchos carecen de la infraestructura y el personal necesarios, aunado a la saturación de otros servicios (p. ej., urgencias) que demandan y consumen los mismos recursos.

Se encontró un dato fundamental: el $20.65 \%$ de los pacientes permanecieron más de 3 días en estancia posoperatoria. Hay que considerar que aquí es donde encontramos a muchos de los pacientes con las complicaciones inmediatas que justifican una estancia hospitalaria más prolongada. Se necesitan estudios centrados en este grupo para definir el porcentaje real de complicaciones en el posquirúrgico inmediato.

En general, está demostrado que la laparoscopia es particularmente ventajosa en los países con ingresos bajos a medios. Los principales retos que enfrentamos son la falta de disponibilidad de equipo, la falta de infraestructura y la falta de personal entrenado. 
Esto nos ha llevado a crear estrategias adaptativas, como el uso de instrumental «autofabricado» o adaptado para usos diferentes de aquellos para los que fueron creados, técnicas asistidas manualmente, nudos extracorpóreos, uso «innovador» de materiales más baratos y el reutilización de material ${ }^{35}$. El gasto en infraestructura, equipamiento, mantenimiento y personal técnico en laparoscopia podría representar una inversión a mediano y largo plazo para apoyar a los servicios de salud rebasados por la necesidad.

Una opción viable y demostrada para mejorar el entrenamiento de los cirujanos, ginecólogos y urólogos es el uso de simuladores (de fábrica y autofabricados) y de modelos animales. El uso de la telemedicina y la telecirugía aún no ha sido explotado, pero podría traer ventajas ${ }^{25}$. En procedimientos asistidos por robot resultan particularmente útiles. Aunque nosotros solo hemos encontrado cinco procedimientos registrados, cada vez serán más comunes. La telecirugía ofrece la posibilidad de realizar procedimientos laparoscópicos que no son comunes en ciertas Entidades Federativas desde Entidades donde son más comunes y por cirujanos con mayor experiencia.

Después de discutir los resultados encontrados, debemos enfatizar la importancia de la gestión de la información en salud. Esta se basa en lograr registros de información de calidad por parte del cirujano, considerando que él es el más interesado y beneficiado en generar conocimiento de sus propias actividades. El registro de calidad implica, por un lado, consistencia y veracidad en la información, y por otro lado un mayor apego a los estándares de atención médica. Una cultura de registro confiable es indispensable para la obtención de información, y su análisis e interpretación generan conocimiento útil para la práctica cotidiana.

\section{Conclusiones}

La aplicación de los abordajes laparoscópicos para diferentes procedimientos quirúrgicos ha ido en aumento en el Sistema de Salud Nacional, aunque todavía le falta diversificarse. Actualmente son mucho más comunes en las mujeres, debido a la mayor incidencia de litiasis vesicular en ellas y a los procedimientos ginecológicos. Hay concordancia con lo reportado en la literatura internacional en relación con los procedimientos más comunes para cirugía general (colecistectomía y apendicectomía), al igual que para ginecología (histerectomía, salpingo-ooforectomía y escisión de ovario). Son pocos los procedimientos laparoscópicos reportados como de corta estancia. Finalmente, se encontró que los procedimientos laparoscópicos en México, aunque seguros, efectivos y aceptados, aún enfrentan retos relacionados con la disponibilidad de elementos organizativos, equipo, infraestructura y entrenamiento, aunque existen diferentes alternativas para vencerlos.

En concreto, en respuesta a la pregunta sobre dónde estamos, podemos decir que seguimos en el proceso de consolidación de la laparoscopia en los sistemas de salud en México mediante Implementación e Implantación. La primera significa ejecutar el proceso involucrándonos activamente como usuario en el desarrollo de lo que se está realizando; la segunda representa una imposición sin considerar nuestra opinión como ejecutores. La respuesta al final, depende de nosotros.

\section{Conflicto de intereses}

Los autores declaran que no existen conflictos de intereses.

\section{Financiamiento}

Se utilizó financiamiento propio de los investigadores.

\section{Responsabilidades éticas}

Protección de personas y animales. Los autores declaran que para esta investigación no se han realizado experimentos en seres humanos ni en animales.

Confidencialidad de los datos. Los autores declaran que han seguido los protocolos de su centro de trabajo sobre la publicación de datos de pacientes.

Derecho a la privacidad y consentimiento informado. Los autores declaran que en este artículo no aparecen datos de pacientes.

\section{Bibliografía}

1. Reddick E. Historia de la colecistectomía laparoscópica. De dónde venimos, dónde estamos, y hacia dónde vamos. Rev Mex Cir Endoscop. 2001;2:36-7.

2. Gutiérrez RL, Grau L. Colecistectomía por laparoscopia: informe del primer caso realizado en México. Endoscopia. 1990;3:99-102.

3. Carrasco JA, Chousleb A, Shuchleib S. Los cambios generados por la cirugía de invasión mínima en la educación quirúrgica. Cir Cir. 2011;79:11-3.

4. Asociación Mexicana de Cirugía General y Consejo Mexicano de Cirugía General. Historia y evolución de la cirugía endoscópica. En: Tratado de cirugía general. 2. ${ }^{\text {a }}$ ed. México, DF: Manual Moderno; 2008. p. 197-9.

5. González V, Marenco CA, Chávez A, González L, Montalvo E. Colecistectomía laparoscópica: resultados de la experiencia del Hospital General de México a nueve años de implementada. Rev Mex Cir Endoscop. 2002;3:71-3.

6. O'Malley E, Boyle E, O'Callaghan A, Coffey C, Walsh S. Role of laparoscopy in penetrating abdominal trauma: a systematic review. World $\mathrm{J}$ Surg. 2013;37:113-22.

7. Karas J. Laparoscopy in non-trauma abdominal emergencies. Eur J Trauma Emerg Surg. 2010;36:10-3. 
8. Tiwari M, Reynoso J, High R, Tsang A, Oleynikov D. Safety, efficacy, and cost-effectiveness of common laparoscopic procedures. Surg Endosc. 2011;24:1127-35.

9. Moria A, Tulandi T. A critical review of laparoscopic total hysterectomy versus laparoscopic supracervical hysterectomy. Gynecol Surg. 2011;8:7-12.

10. Pearl J, Price R, Richardson W, Fanelli R. Guidelines for diagnosis, treatment, and use of laparoscopy for surgical problems during pregnancy. Surg Endosc. 2011;25:3479-92.

11. Mazón J, Moreno M, Magos F, Rojano M, Palacios JA, Herrera JJ. Cirugía endoscópica ambulatoria. 2004. (Consultado el 2 de julio de 2016.) Disponible en: http://www.ejournal.unam.mx/rfm/no47-4/RFM47403.pdf

12. López G, Zavalza JF. Paipilla O, Lee S. Colecistectomías laparoscópicas realizadas en unidad de cirugía ambulatoria. Cir Gen. 2011;33:104-9.

13. Tuschy B, Berlit S, Sütterlin M, Hornemann A. Duration of hospital stay after gynaecologic laparoscopic surgery: preferences of patients. Arch Gynecol Obstet. 2013;288:335-9.

14. Rocha E, Quijano O, Cortés G, López D, Abrego J, Gómez J. Tratamiento laparoscópico del cáncer de endometrio. Experiencia institucional. Gac Mex Onc. 2015;14:8-35.

15. Sánchez GM. Indicadores de gestión hospitalaria. Rev Inst Nal Enf Resp Mex. 2005;18:132-41

16. Gómez DG, Sesma S, Becerril VM, Knaul FM, Arreola H, Frenk J. Sistema de salud de México. Salud Pública Mex. 2011;53:S220-32.

17. Priego P, Ramiro C, Molina J, Rodríguez G, Lobo E, Galindo J, et al. Results of laparoscopic cholecystectomy in a third-level university hospital after 17 years of experience. Rev Enferm Dig. 2009;101:20-30.

18. Szabo K, Rothe A, Shamiyeh A. Laparoscopic cholecystectomy - review over 20 years with attention on acute cholecystitis and conversion. Eur Surg. 2012;44:28-37.

19. Litwin D, Cahan M. Laparoscopic cholecystectomy. Surg Clin N Am. 2008;88:295-313.

20. Guller U, Hervey S, Purves H, Muhlbaier L, Eubanks S, Pietrobon R Laparoscopic versus open appendectomy. Outcomes comparison based on a large administrative database. Ann Surg. 2004;239:43-52.

21. Hernias de la pared abdominal. Tratamiento actual. Mayagoitia González, J. Alfil. 2a. Edición, Ciudad de México, México, 2009, pp. 395-407.

22. Edwards CC II, Bailey RW. Laparoscopic hernia repair: the learning curve. Surg Laparosc Endosc Percutan Tech. 2000;10:149-53.
23. McCormack K, Wake B, Pérez J, Fraser C, Cook J, Mclntosh E, et al. Laparoscopic surgery for inguinal hernia repair: systematic review of effectiveness and economic evaluation. Health Technol Assess. 2005;9:1-203.

24. Secretaría de Salud. MH 2015: Mejores Hospitales de la Secretaría de Salud Federal y los Servicios Estatales de Salud. Revista de la Dirección General de Evaluación del Desempeño. 2015;1:12-3.

25. Norma Oficial Mexicana NOM-040-SSA2-2004, en materia de información en salud. Diario Oficial de la Federación, 28 de septiembre de 2005.

26. Moria A, Tulandi T. A critical review of laparoscopic total hysterectomy versus laparoscopic supracervical hysterectomy. Gynecol Surg. 2011;14:28-35.

27. Bedaiwy M, Sharp H, Falcone T. Hurd W. Gynecologic laparoscopy. Gynecologic laparoscopic in clinical reproductive medicine and surgery: a practical guide. Springer Science Business Media. Durham, North Carolina, EUA, 2011.

28. Campos JG, Bravo E, Sedano A, Torres J, Hernández G, Estrada C, et al. Experiencia de nefrectomía laparoscópica en el Hospital Central Militar. Rev Mex Urol. 2013;73:72-6.

29. Yoon PD, Pang T, Siriwardhane M, Richardson A, Hollands M, Pleass H, et al. Laparoscopic partial cholecystectomy: a way of getting out of trouble. Int J Hepatobiliary Pancreat Dis. 2016;6:68-75.

30. Albright JB, Fakhre GP, Nields WW, Metzger PP. Incidental appendectomy: 18-year pathologic survey and cost effectiveness in the nonmanaged-care setting. J Am Coll Surg. 2007;205:298-306.

31. Dumon K, Murayama K. Bariatric surgery outcomes. Surg Clin N Am. 2011;91:1313-38.

32. López F, Herrera M. El papel actual del tratamiento quirúrgico. ¿Puede la cirugía curar enfermedades metabólicas? Bol Med Hosp Infant Mex. 2008:65:568-78.

33. Mason R, Moazzez A, Moroney J, Katkhouda N. Laparoscopic vs open appendectomy in obese patients: outcomes using the American College of Surgeons National Surgical Quality Improvement Program Database. J Am Coll Surg. 2012;215:88-99.

34. Romero C. Cirugía metabólica en América Latina. Cir Gen. 2010;32:121-4.

35. Tiwari M, Reynoso J, High R, Tsang A, Oleynikov D. Safety, efficacy, and cost-effectiveness of common laparoscopic procedures. Surg Endosc. $2010 ; 25: 1127-35$. 